\title{
A BOUND ON THE RATE OF CONVERGENCE IN THE CENTRAL LIMIT THEOREM FOR RENEWAL PROCESSES UNDER SECOND MOMENT CONDITIONS
}

\author{
Gesine Reinert and Ce Yang*
}

November 9, 2018

\begin{abstract}
A famous result in renewal theory is the Central Limit Theorem for renewal processes. As in applications usually only observations from a finite time interval are available, a bound on the Kolmogorov distance to the normal distribution is desirable. Here we provide an explicit non-uniform bound for the Renewal Central Limit Theorem based on Stein's method and track the explicit values of the constants. For this bound the inter-arrival time distribution is required to have only a second moment. As an intermediate result of independent interest we obtain explicit bounds in a non-central Berry-Essén theorem under second moment conditions.
\end{abstract}

Keywords: Rate of Convergence; Central Limit Theorem; Stein's Method AMS Subject Classification: 60F0560G50

\section{Introduction}

Let $Z, Z_{i}, i=1,2, \ldots$ be i.i.d. non-negative random variables with positive mean $\mu$ and finite variance $\sigma^{2}$, and let

$$
X_{t}=\max \left\{n: \sum_{i=1}^{n} Z_{i} \leq t\right\} .
$$

Then $\left(X_{t}, t \geq 0\right)$ is a classical renewal process.

Renewal processes are a cornerstone in applied probability and appear in a number of applications, see for example [8] and references therein. As in applications, time is finite, a quantification of the the rate of convergence

\footnotetext{
${ }^{*}$ Oxford University; reinert@stats.ox.ac.uk
} 
to normal is desirable. Also note that $X_{t}$ only takes on values in $\{0,1, \ldots\}$. In [6] it is shown that when $\gamma:=\mathbb{E}\left(|Z-\mu|^{3}\right)<\infty$ then

$$
\sup _{n=0,1, \ldots}\left|\mathbb{P}\left(X_{t}<n\right)-\Phi\left(\frac{(n \mu-t) \sqrt{\mu}}{\sigma \sqrt{t}}\right)\right| \leq 4\left(\frac{\gamma}{\sigma}\right)^{3}\left(\frac{\sqrt{\mu}}{\sqrt{t}}\right)^{\frac{1}{2}}
$$

where $\Phi$ is the c.d.f. of the standard normal distribution. Also in [6] a similar bound is indicated when $Z$ possesses moments of order $\alpha$ for some $2<\alpha<3$. Under the third moment assumption, this bound was generalised to the bivariate case in [1], which in turn was generalised to a $k$-variate process in [7. The result was extended in [9] to allow for non-identically distributed inter-arrival times $Z_{i}$, again under third moment assumptions. In [2], Theorem 17.3, a functional central limit theorem for the renewal process is shown. In particular, as $t \rightarrow \infty, X_{t}$ is asymptotically normally distributed with mean $\frac{t}{\mu}$ and variance $\frac{\sigma^{2} t}{\mu^{3}}$. Hence second moments suffice for the normal approximation. Unfortunately [2] does not give a bound on the rate of convergence.

In this paper we provide a bound on the rate of convergence in the case that $Z$ has only second moments; this bound is of the order $t^{-\frac{1}{2}}$. As an intermediate result we provide explicit constants for a non-uniform BerryEsseén theorem, quantifying Theorem 2.2 in [4] (also Theorem 8.1 in [3]). Our main tool is Stein's method.

The paper is organised as follows. In Section 2 we introduce notation, we give some bounds on the tail of the normal distribution, and we provide some background from Stein's method. Section 3 gives the main result, with a proof. The proof is based on the approach to obtain non-uniform bound from sums of i.i.d. random variables in Chapter 8 of [3], while deriving explicit bounds for the required intermediary results from that chapter. Proofs of auxiliary results are given in Section 4. For convenience, in the Appendix we re-state results from [3] which are used in this paper.

\section{Notations, tail bounds, and results from Stein's method}

\section{$2.1 \quad$ Notations}

Let $Z_{n}, n \geq 0$, be independent identically distributed, positive random variables. Let $T_{n}=Z_{0}+\ldots+Z_{n-1}, n \geq 1$. The process $X=\left(X_{t}, t \geq 0\right)$ defined by $X_{t}=\#\left\{n \geq 1: T_{n} \leq t\right\}$ is the renewal process of interest. 
For a renewal process $X_{t}$ whose inter-arrival times $Z_{i}$ have mean $\mu$ and variance $\sigma^{2}$, and $n, t \in\{0,1, \ldots$,$\} fixed, we aim to compare \mathbb{P}\left(X_{t} \leq n\right)=$ $\mathbb{P}\left(\frac{X_{t}-\frac{t}{\mu}}{\sigma \sqrt{t} \mu^{-\frac{3}{2}}} \leq \frac{(n \mu-t) \sqrt{\mu}}{\sigma \sqrt{t}}\right)$ to $\Phi\left(\frac{(n \mu-t) \sqrt{\mu}}{\sigma \sqrt{t}}\right)$.

\subsection{Normal tail bounds}

The following results will be useful when we develop the bounds. Firstly, for every $w>0$, the standard normal tail bound

$$
\frac{1}{4\left(1+w^{2}\right)} e^{-\frac{w^{2}}{2}} \leq \Phi(-w)=1-\Phi(w) \leq \min \left(\frac{1}{2}, \frac{1}{w \sqrt{2 \pi}}\right) e^{-\frac{w^{2}}{2}}
$$

holds. This is a well-known result, see for example Inequality (2.11) and p.243 in [3]. The next result assesses the smoothness of the standard normal c.d.f., as follows.

Lemma 2.1. For $\mu>0, \sigma>0, n \geq 1$ and $t>0$, let

$$
I=\left|\Phi\left(\frac{n \mu-t}{\sigma \sqrt{n}}\right)-\Phi\left(\frac{(n \mu-t) \sqrt{\mu}}{\sigma \sqrt{t}}\right)\right|
$$

Then

$$
I \leq \begin{cases}\frac{\sqrt{2}}{e \sqrt{\pi}} \frac{\sigma}{\sqrt{t \mu}} & \text { if } t \leq n \mu ; \\ \frac{16}{e^{2} \sqrt{2 \pi}} \frac{t^{2} \sigma^{3}}{n^{\frac{1}{2}} \mu^{2}(t-n \mu)^{2}(\sqrt{n \mu t}+t)} & \text { if } t>n \mu .\end{cases}
$$

A proof of Lemma 2.1] is in Section 4 .

\subsection{Results from Stein's method}

Stein's method, origating from [10] is a powerful tool to assess distances between distributions. The proof of the statements below can be found in 3], pp.13-16. Let $W$ be a random variable and suppose that the aim is to bound $|\mathbb{P}(W \leq z)-\Phi(z)|$ for all real $z$. For fixed $z \in \mathbb{R}$, the unique bounded solution $f(w):=f_{z}(w)$ of the so-called Stein equation

$$
f^{\prime}(w)-w f(w)=\mathbb{1}(w \leq z)-\Phi(z)
$$

is given by

$$
f_{z}(w)= \begin{cases}\sqrt{2 \pi} e^{w^{2} / 2} \Phi(w)[1-\Phi(z)] & \text { if } w \leq z \\ \sqrt{2 \pi} e^{w^{2} / 2} \Phi(z)[1-\Phi(w)] & \text { if } w>z\end{cases}
$$


With this solution,

$$
\mathbb{P}(W \leq z)-\Phi(z)=\mathbb{E}\left\{f^{\prime}(W)-W f(W)\right\}
$$

and the right-hand side depends only on the distribution of $W$ and can often be bounded using Taylor expansion. Moreover, for the solution $f_{z}$ of the Stein equation (3), $w f_{z}(w)$ in an increasing function of $w$, and for all real $w$,

$$
\begin{aligned}
\left|f_{z}^{\prime}(w)\right| & \leq 1 \\
0<f_{z}(w) & \leq \min \left(\frac{\sqrt{2 \pi}}{4}, \frac{1}{|z|}\right) .
\end{aligned}
$$

\section{A non-uniform bound for the Renewal Central Limit Theorem}

Our main result is Theorem 3.1. As $\mathbb{P}\left(X_{t} \leq n\right)=0$ for $n<1$, we restrict attention to the regime that $n \geq 1$.

Theorem 3.1 (Bound for the Renewal Central Limit Theorem Under Second Moment Assumptions). Let $X=\left(X_{t}, t \geq 0\right)$ be a renewal process whose inter-arrival times $Z_{n}, n \geq 0$, have finite mean $\mu \in(0, \infty)$ and finite variance $\sigma^{2} \in(0, \infty)$. Then for $n \geq 1$,

$$
\begin{aligned}
& \left|\mathbb{P}\left(X_{t} \leq n\right)-\Phi\left(\frac{(n \mu-t) \sqrt{\mu}}{\sigma \sqrt{t}}\right)\right| \\
& \leq \mathbb{1}(t \leq n \mu) \frac{\sqrt{2}}{e \sqrt{\pi}} \frac{\sigma}{\sqrt{t} \mu}+\mathbb{1}(t>n \mu) \frac{32}{e^{2} \sqrt{2 \pi}} \frac{1}{\sqrt{t}}\left(\frac{\sigma^{3}}{\mu^{2} \sqrt{t}}+\frac{\sigma}{(224)^{2} \sqrt{\mu}}\right) \\
& \quad+50,990\left(1+\left|\frac{t-n \mu}{\sigma \sqrt{n}}\right|\right)^{-2} .
\end{aligned}
$$

Before we prove this results, here are some remarks.

Remark 3.2. $\quad$ 1. The explicit value of the constant in Theorem 3.1 is large. This is because the calculation of the constant is not optimized. As a result, the bound is not informative for small values of $n$.

2. The bound is the order of $t^{-\frac{1}{2}}$. The bound deteriorates for $t$ close to the expectation $n \mu$. 
3. Theorem 3.1 does not assume the existence of the finite third moments. It holds as long as the inter-arrival times have finite variance. This result enables us to assess the rate of convergence in the Central Limit Theorem for example for a renewal process whose inter-arrival times $Z_{i}$ follow a Pareto Pareto $(n, \alpha)$-distribution with $\alpha \in[2,3)$ for $i \geq 1$.

For the proof of Theorem [3.1, recall that $T_{n}=\sum_{i=1}^{n} Z_{i}$ has mean $n \mu$ and variance $n \sigma^{2}$, and $\mathbb{P}\left(X_{t} \leq n\right)=\mathbb{P}\left(T_{n} \geq t\right)$. Moreover, the standardised $T_{n}$ satisfies the Central Limit Theorem. We decompose

$$
\begin{aligned}
\mathbb{P}\left(X_{t} \leq n\right)-\Phi\left(\frac{(n \mu-t) \sqrt{\mu}}{\sigma \sqrt{t}}\right)= & \mathbb{P}\left(T_{n} \geq t\right)-\left\{1-\Phi\left(\frac{t-n \mu}{\sigma \sqrt{n}}\right)\right\} \\
& +\Phi\left(\frac{n \mu-t}{\sigma \sqrt{n}}\right)-\Phi\left(\frac{(n \mu-t) \sqrt{\mu}}{\sigma \sqrt{t}}\right) .
\end{aligned}
$$

We bound the terms (8) and (9) separately. For (9) we employ the tail bounds for the normal distribution from Lemma 2.1. For (86) we derive nonuniform bounds using ideas from Chapter 8 in [3] - our Theorem 3.3 is a version of Theorem 8.1 in [3] but with the constants in the bound made explicit. This bound is of interest in their own right and hence we give it as a theorem.

Theorem 3.3. Let $\xi_{1}, \xi_{2}, \ldots \xi_{n}$ be i.i.d. random variables with mean $\mu$ and variance $\sigma^{2}$. Let $W$ denote their sum, $W=\sum_{i=1}^{n} \xi_{i}$. Let

$$
\beta_{2}=\sum_{i=1}^{n} \mathbb{E} \xi_{i}^{2} \mathbb{1}\left(\left|\xi_{i}\right|>1\right) \text { and } \beta_{3}=\sum_{i=1}^{n} \mathbb{E}\left|\xi_{i}\right|^{3} \mathbb{1}\left(\left|\xi_{i}\right| \leq 1\right) .
$$

Then, for all $z \in \mathbb{R}$,

$$
|\mathbb{P}(W \leq z)-\Phi(z)| \leq 2 \sum_{i=1}^{n} \mathbb{P}\left(\left|\xi_{i}\right|>\frac{1 \vee|z|}{4}\right)+C_{2}(1+|z|)^{-2}\left(\beta_{2}+\beta_{3}\right),
$$

where

$$
C_{2} \leq \begin{cases}15 & \text { if } \beta_{2}+\beta_{3} \geq 1 \\ 37 & \text { if } \beta_{2}+\beta_{3}<1 \text { and }|z| \leq 2 \\ 25431 & \text { if } \beta_{2}+\beta_{3}<1 \text { and }|z|>2\end{cases}
$$

The proof of Theorem 3.3 is found in Section 4, The proof of Theorem 3.1 is now almost immediate. 
Proof of Theorem 3.1. First, Term (9) is bounded directly in Lemma 2.1. The bound arising from (8) is less than 1 only when

$$
\frac{|t-n \mu|}{\sigma \sqrt{n}} \geq \sqrt{50,990}-1
$$

Hence if $\frac{|t-n \mu|}{\sigma \sqrt{n}} \leq 224$, the claim is trivially true. So we apply Lemma 2.1 for $\frac{|t-n \mu|}{\sigma \sqrt{n}}>224$, which turns the non-uniform bound for the regime $t>n \mu$ and $n \geq 1$ into a uniform bound for the regime $\frac{t-n \mu}{\sigma \sqrt{n}}>224$, for which it holds that

$$
\frac{t}{t-n \mu} \leq 1+\frac{n \mu}{224 \sigma \sqrt{n}}
$$

so that

$$
\frac{16}{e^{2} \sqrt{2 \pi}} \frac{t^{2} \sigma^{3}}{\sqrt{n} \mu^{2}(t-n \mu)^{2}(\sqrt{n \mu t}+t)} \leq \frac{32}{e^{2} \sqrt{2 \pi}} \frac{1}{\sqrt{t}}\left(\frac{\sigma^{3}}{\mu^{2} \sqrt{t}}+\frac{\sigma}{(224)^{2} \sqrt{\mu}}\right) .
$$

This gives the first part of the bound.

For Term (8), using Theorem 3.3 it remains to show that

$$
2 \sum_{i=1}^{n} \mathbb{P}\left(\left|\xi_{i}\right|>\frac{1 \vee|z|}{4}\right) \leq 128\left(\frac{1}{1+|z|}\right)^{2}
$$

with $\xi=\frac{Z_{i}-\mu}{\sigma \sqrt{n}}$ and then apply this inequalty to $z=\left|\frac{t-n \mu}{\sigma \sqrt{n}}\right|$. Note that $\frac{1+|z|}{2} \leq 1 \vee|z|$. So, using Markov's inequality,

$$
\begin{aligned}
\sum_{i=1}^{n} \mathbb{P}\left(\left|\xi_{i}\right|>\frac{1 \vee|z|}{4}\right) & \leq \sum_{i=1}^{n} \mathbb{P}\left(\left|\xi_{i}\right|>\frac{1+|z|}{8}\right) \\
& \leq\left(\frac{8}{1+|z|}\right)^{2} \sum_{i=1}^{n} \mathbb{E} \xi_{i}^{2}=\left(\frac{8}{1+|z|}\right)^{2} .
\end{aligned}
$$

Setting $C=128+2 C_{2}$ gives the assertion.

Remark 3.4. With the notation from Theorem 3.3, under the same assumptions as for Theorem [3.1, using Theorem 3.3 in [5] with $\xi_{i}=\frac{Z_{i}-\mu}{\sigma \sqrt{n}}$ to bound (9) gives the bound

$$
\begin{aligned}
& \left|\mathbb{P}\left(X_{t} \leq n\right)-\Phi\left(\frac{(n \mu-t) \sqrt{\mu}}{\sigma \sqrt{t}}\right)\right| \\
& \leq \frac{1}{\sqrt{t}} \max \left\{\frac{\sqrt{2}}{e \sqrt{\pi}} \frac{\sigma}{\mu}, \frac{32}{e^{2} \sqrt{2 \pi}}\left(\frac{\sigma^{3}}{\mu^{2} \sqrt{t}}+\frac{\sigma}{(224)^{2} \sqrt{\mu}}\right)\right\}+4\left(4 \beta_{2}+3 \beta_{3}\right) .
\end{aligned}
$$


The terms $\beta_{2}$ and $\beta_{3}$ depend on $n$ as well as on $t$ in an implicit fashion but may be straightforward to calculate in some situations.

\section{Remaining proofs of results}

Proof of Lemma 2.1. To bound $I=\left|\Phi\left(\frac{n \mu-t}{\sigma \sqrt{n}}\right)-\Phi\left(\frac{(n \mu-t) \sqrt{\mu}}{\sigma \sqrt{t}}\right)\right|$ we consider two cases.

Case 1: $n \mu \geq t$ : If $t \leq n \mu$ then $\frac{n \mu}{t} \geq 1$ and

$$
\begin{aligned}
I & \leq \frac{1}{\sqrt{2 \pi}} \frac{n \mu-t}{\sigma \sqrt{n}}\left(\frac{\sqrt{n \mu}}{\sqrt{t}}-1\right) \exp \left\{-\frac{1}{2}\left(\frac{n \mu-t}{\sigma \sqrt{n}}\right)^{2}\right\} \\
& \leq \frac{1}{\sqrt{2 \pi}} \frac{\sigma \sqrt{n}}{t+\sqrt{t n \mu}} \sup _{x \geq 0}\left\{x^{2} e^{-\frac{1}{2} x^{2}}\right\} \\
& \leq \frac{\sqrt{2}}{e \sqrt{\pi}} \frac{\sigma}{\sqrt{t \mu}}
\end{aligned}
$$

Case 2: $t>n \mu$ : If $t>n \mu$ then $\frac{n \mu}{t}<1$ and

$$
\begin{aligned}
I & \leq \frac{1}{\sqrt{2 \pi}} \frac{t-n \mu}{\sigma \sqrt{n}}\left(1-\frac{\sqrt{n \mu}}{\sqrt{t}}\right) \exp \left\{-\frac{1}{2}\left(\frac{(t-n \mu)}{\sigma \sqrt{n}} \frac{\sqrt{n \mu}}{\sqrt{t}}\right)^{2}\right\} \\
& \leq \frac{1}{\sqrt{2 \pi}} \frac{t^{2} \sigma^{3} n^{\frac{3}{2}}}{(n \mu)^{2}(t-n \mu)^{2}(\sqrt{n \mu t}+t)} \sup _{x \geq 0}\left\{x^{4} e^{-\frac{1}{2} x^{2}}\right\} \\
& \leq \frac{16}{e^{2} \sqrt{2 \pi}} \frac{t^{2} \sigma^{3}}{n^{\frac{1}{2}} \mu^{2}(t-n \mu)^{2}(\sqrt{n \mu t}+t)} .
\end{aligned}
$$

This completes the proof.

\section{Proof of Theorem 3.3}

For the proof of Theorem 3.3 we first show an auxiliary result, Lemma 4.1. which gives an explicit bound for Lemma 8.4 in [3].

Let $\xi_{1}, \ldots, \xi_{n}$ denote independent random variables with zero means and variances summing to one. Let $W$ denote their sum, $W=\sum_{i=1}^{n} \xi_{i}$. We consider the truncated random variables and their sums

$$
\bar{x}_{i}=\xi_{i} \mathbb{1}\left(\xi_{i} \leq 1\right), \quad \bar{W}=\sum_{i=1}^{n} \bar{x}_{i}, \text { and } \bar{W}^{(i)}=\bar{W}-\bar{x}_{i} .
$$


Lemma 4.1. Let $f_{z}$ denote the solution to the Stein Equation (3). For $z>2$ and for all $s \leq t \leq 1$, we have

$$
\begin{aligned}
& \mathbb{E}\left[\left(\bar{W}^{(i)}+\bar{x}_{i}\right) f_{z}\left(\bar{W}^{(i)}+\bar{x}_{i}\right)-\left(\bar{W}^{(i)}+t\right) f_{z}\left(\bar{W}^{(i)}+t\right)\right] \\
& \leq\left(25.8+\frac{20 e^{e^{2}-2}}{\sqrt{2 \pi}}\right) e^{-\frac{z}{2}} \min (1,|s|+|t|) .
\end{aligned}
$$

Proof of Lemma 4.1. Let $g(w)=\left(w f_{z}(w)\right)^{\prime}$. Then for all $s \leq t \leq 1$,

$$
\left.\mathbb{E}\left(\bar{W}^{(i)}+t\right) f_{z}\left(\bar{W}^{(i)}+t\right)-\left(\bar{W}^{(i)}-s\right) f_{z}\left(\bar{W}^{(i)}-s\right)\right]=\int_{s}^{t} \mathbb{E} g\left(\bar{W}^{(i)}+u\right) d u .
$$

Using (4), we can compute that

$$
g(w)= \begin{cases}\sqrt{2 \pi}(1-\Phi(z))\left(\left(1+w^{2}\right) e^{w^{2} / 2} \Phi(w)+\frac{w}{\sqrt{2 \pi}}\right) & \text { if } w \leq z \\ \sqrt{2 \pi} \Phi(z)\left(\left(1+w^{2}\right) e^{w^{2} / 2}(1-\Phi(w))-\frac{w}{\sqrt{2 \pi}}\right) & \text { if } w>z\end{cases}
$$

Instead of $w \leq z$, we consider whether or not $w \leq \frac{z}{2}$. We split the problem into four cases.

Case 1. If $w \leq 0$, then (5.4) from [4] gives

$$
\sqrt{2 \pi}\left(1+w^{2}\right) e^{w^{2} / 2} \Phi(w)+w \leq \frac{2}{1+|w|^{3}} \quad \text { for } w \leq 0 .
$$

In this case, $w \leq 0<z$, so

$$
g(w) \leq(1-\Phi(z)) \frac{2}{1+|w|^{3}} \leq \frac{4\left(1+z^{2}\right)\left(1+z^{3}\right)}{1+|w|^{3}} e^{\frac{z^{2}}{8}}(1-\Phi(z)) .
$$

Case 2. If $0<w \leq \frac{z}{2}$, then

$$
\begin{aligned}
g(w) & \leq(1-\Phi(z))\left(3\left(1+z^{2}\right) e^{\frac{z^{2}}{8}}+z\right) \\
& \leq \frac{4\left(1+z^{2}\right)\left(1+z^{3}\right)}{1+|w|^{3}} e^{\frac{z^{2}}{8}}(1-\Phi(z)) .
\end{aligned}
$$

Case 3. If $\frac{z}{2}<w \leq z$, then

$$
\begin{aligned}
g(w) & \leq \sqrt{2 \pi}(1-\Phi(z))\left(\left(1+z^{2}\right) e^{z^{2} / 2}+\frac{z}{\sqrt{2 \pi}}\right) \\
& \leq 8\left(1+z^{2}\right) e^{z^{2} / 2}(1-\Phi(z))
\end{aligned}
$$


Case 4. If $z<w$, then replacing $w$ by $-w$ in (20) gives

$$
\sqrt{2 \pi}\left(1+w^{2}\right) e^{w^{2} / 2} \Phi(-w)-w \leq \frac{2}{1+|w|^{3}} .
$$

In this case, we use the standard normal tail bound (2) to obtain

$$
g(w) \leq \Phi(z) \frac{2}{1+|w|^{3}} \leq 2=8\left(1+z^{2}\right) e^{\frac{z^{2}}{2}} \frac{e^{-z^{2} / 2}}{4\left(1+z^{2}\right)} \leq 8\left(1+z^{2}\right) e^{\frac{z^{2}}{2}}(1-\Phi(z)) .
$$

Collecting (21), (22), (23) and (24),

$$
g(w) \leq \begin{cases}\frac{4\left(1+z^{2}\right)\left(1+z^{3}\right)}{1+|w|^{3}} e^{\frac{z^{2}}{8}}(1-\Phi(z)) & \text { if } w \leq \frac{z}{2} \\ 8\left(1+z^{2}\right) e^{\frac{z^{2}}{2}}(1-\Phi(z)) & \text { if } w>\frac{z}{2}\end{cases}
$$

So for any $u \in[s, t]$, since $z>2$, we have

$$
\begin{aligned}
\mathbb{E} g\left(\bar{W}^{(i)}+u\right)= & \mathbb{E}\left[g\left(\bar{W}^{(i)}+u\right) \mathbb{1}_{\bar{W}^{(i)}+u \leq \frac{z}{2}}\right]+\mathbb{E}\left[g\left(\bar{W}^{(i)}+u\right) \mathbb{1}_{\bar{W}^{(i)}+u>\frac{z}{2}}\right] \\
\leq & \mathbb{E}\left[\frac{1}{1+\left|\bar{W}^{(i)}+u\right|^{3}}\right] 4\left(1+z^{2}\right)\left(1+z^{3}\right) e^{\frac{z^{2}}{8}}(1-\Phi(z)) \\
& +8\left(1+z^{2}\right) e^{\frac{z^{2}}{2}}(1-\Phi(z)) \mathbb{P}\left(\bar{W}^{(i)}+u>\frac{z}{2}\right) \\
\leq & \mathbb{E}\left[\frac{1}{1+\left|\bar{W}^{(i)}+u\right|^{3}}\right] 4\left(1+z^{2}\right)\left(1+z^{3}\right) e^{\frac{z^{2}}{8}} \frac{1}{z \sqrt{2 \pi}} e^{-\frac{z^{2}}{2}} \\
& +8\left(1+z^{2}\right) e^{\frac{z^{2}}{2}} \frac{1}{z \sqrt{2 \pi}} e^{-\frac{z^{2}}{2}} \mathbb{P}\left(e^{2 u} e^{2 \bar{W}^{(i)}}>e^{z}\right) .
\end{aligned}
$$

Using Markov's Inequality, since $u \leq t \leq 1$, we obtain

$$
\begin{aligned}
\mathbb{E} g\left(\bar{W}^{(i)}+u\right) & \leq \frac{4\left(1+z^{2}\right)\left(1+z^{3}\right) e^{-\frac{3 z^{2}}{8}}}{z \sqrt{2 \pi}} \mathbb{E}\left[\frac{1}{1+\left|\bar{W}^{(i)}+u\right|^{3}}\right] \\
& +\frac{8\left(1+z^{2}\right)}{z \sqrt{2 \pi}} e^{2 u-z} \mathbb{E}\left[e^{2 \bar{W}^{(i)}}\right] \\
& \leq \frac{4\left(1+z^{2}\right)\left(1+z^{3}\right) e^{-\frac{3 z^{2}}{8}}}{z \sqrt{2 \pi}}+\frac{8\left(1+z^{2}\right)}{z \sqrt{2 \pi}} e^{2} e^{-z} e^{e^{2}-3} \\
& \leq\left(25.8+\frac{20}{\sqrt{2 \pi}} e^{e^{2}-2}\right) e^{-\frac{z}{2}}
\end{aligned}
$$


where we used Lemma 8.2 from [3] with $t=2$ and $\alpha=B=1$. So for $z>2$, from (26) we have

$$
\begin{aligned}
\int_{s}^{t} \mathbb{E} g\left(\bar{W}^{(i)}+u\right) d u & \leq\left(25.8+\frac{20 e}{\sqrt{2 \pi}} e^{e^{2}-3}\right) e^{-\frac{z}{2}}(t-s) \\
& \leq\left(25.8+\frac{20 e^{e^{2}-2}}{\sqrt{2 \pi}}\right) e^{-\frac{z}{2}}(|t|+|s|) .
\end{aligned}
$$

The assertion follows.

Proof of Theorem 3.3. Note that it is enough to consider $z \geq 0$. To see this, replacing $W$ by $-W$ gives

$$
|\mathbb{P}(-W \leq z)-\Phi(z)|=|\mathbb{P}(-W \geq z)-\Phi(-z)|=|\mathbb{P}(W \leq-z)-\Phi(-z)| .
$$

The case $\beta_{2}+\beta_{3} \geq 1$

We start with the case of $\beta_{2}+\beta_{3} \geq 1$. Note that

$$
|\mathbb{P}(W \leq z)-\Phi(z)|=|\mathbb{P}(W>z)-(1-\Phi(z))| \leq \mathbb{P}(W>z)+1-\Phi(z) .
$$

As $W$ is sum of independent random variables with zero means and variances less than or equal to one, we apply Lemma 8.1 in [3] with $B=1$ and $p=2$ to obtain

$$
\begin{aligned}
\mathbb{P}(W \geq z) & \leq \mathbb{P}\left(\max _{1 \leq i \leq n}\left|\xi_{i}\right|>\frac{z \vee 1}{2}\right)+e^{2}\left(1+\frac{z^{2}}{2}\right)^{-2} \\
& \leq \sum_{i=1}^{n} \mathbb{P}\left(\left|\xi_{i}\right|>\frac{z \vee 1}{4}\right)+e^{2}\left(1+\frac{z^{2}}{2}\right)^{-2}
\end{aligned}
$$

To write (29) as a bound of the form (10), we bound $e^{2}\left(1+\frac{z^{2}}{2}\right)^{-2}$ by $1.867 e^{2}(1+z)^{-2}$.

For $1-\Phi(z)$ we apply the standard normal tail bound (2) and obtain

$$
\begin{aligned}
|\mathbb{P}(W \leq z)-\Phi(z)| \leq & \mathbb{P}(W \geq z)+|1-\Phi(z)| \\
\leq & \sum_{i=1}^{n} \mathbb{P}\left(\left|\xi_{i}\right|>\frac{z \vee 1}{4}\right)+1.867 e^{2}(1+z)^{-2}\left(\beta_{2}+\beta_{3}\right) \\
& +\min \left(\frac{1}{2}, \frac{1}{z \sqrt{2 \pi}}\right) e^{-\frac{z^{2}}{2}}
\end{aligned}
$$


Now we bound the standard normal tail bound in (30) by

$$
\min \left(\frac{1}{2}, \frac{1}{z \sqrt{2 \pi}}\right) e^{-\frac{z^{2}}{2}} \leq 1.176(1+z)^{-2} .
$$

Substituting (31) into (30) gives that for $z \geq 0$,

$$
\begin{aligned}
& |\mathbb{P}(W \leq z)-\Phi(z)| \\
& \leq \sum_{i=1}^{n} \mathbb{P}\left(\left|\xi_{i}\right|>\frac{z \vee 1}{4}\right)+\left(1.867 e^{2}+1.176\right)(1+z)^{-2}\left(\beta_{2}+\beta_{3}\right) \\
& \leq 2 \sum_{i=1}^{n} \mathbb{P}\left(\left|\xi_{i}\right|>\frac{1 \vee|z|}{4}\right)+\left(1.867 e^{2}+1.176\right)(1+|z|)^{-2}\left(\beta_{2}+\beta_{3}\right) .
\end{aligned}
$$

Since $1.867 e^{2}+1.176<15$, we have proved the theorem for the case that $\beta_{2}+\beta_{3} \geq 1$.

The case $\beta_{2}+\beta_{3}<1$ and $z \leq 2$

Next, we consider the case of $\beta_{2}+\beta_{3}<1$. We distinguish whether or not $z>2$.

If $z \in[0,2]$, then we use the uniform bound (3.31) from [3], which states that

$$
\sup _{z \in \mathbb{R}}|\mathbb{P}(W \leq z)-\Phi(z)| \leq 4.1\left(\beta_{2}+\beta_{3}\right)
$$

We bound 4.1 by $37(1+|z|)^{-2}$ for $z \in[0,2]$ because $4.1 \times(1+2)^{2}<37$. So we have

$$
|\mathbb{P}(W \leq z)-\Phi(z)| \leq 2 \sum_{i=1}^{n} \mathbb{P}\left(\left|\xi_{i}\right|>\frac{1 \vee|z|}{4}\right)+37(1+|z|)^{-2}\left(\beta_{2}+\beta_{3}\right) .
$$

Thus we have proved the theorem when $\beta_{2}+\beta_{3}<1$ and $z \in[0,2]$.

The case $\beta_{2}+\beta_{3}<1$ and $z>2$

Our remaining task is to prove the theorem when $\beta_{2}+\beta_{3}<1$ and $z>2$. Recall the notations $\bar{x}_{i}=\xi_{i} \mathbb{1}_{\xi_{i} \leq 1}, \bar{W}=\sum_{i=1}^{n} \bar{x}_{i}$, and $\bar{W}^{(i)}=\bar{W}-\bar{x}_{i}$. The idea is to show that $\mathbb{P}(W>z)$ is close to $\mathbb{P}(\bar{W}>z)$ for $z>2$. Observing that

$$
\begin{aligned}
\{W>z\} & =\left\{W>z, \max _{1 \leq i \leq n} \xi_{i}>1\right\} \cup\left\{W>z, \max _{1 \leq i \leq n} \xi_{i} \leq 1\right\} \\
& \subset\left\{W>z, \max _{1 \leq i \leq n} \xi_{i}>1\right\} \cup\{\bar{W}>z\}
\end{aligned}
$$


and $W \geq \bar{W}, \mathbb{P}(\bar{W}>z)$ yields

$$
\mathbb{P}(\bar{W}>z) \leq \mathbb{P}(W>z) \leq \mathbb{P}(\bar{W}>z)+\mathbb{P}\left(W>z, \max _{1 \leq i \leq n} \xi_{i}>1\right) .
$$

From Lemma 8.3 in [3], with $p=2$ and $z>2$,

$$
\mathbb{P}\left(W \geq z, \max _{1 \leq i \leq n} \xi_{i}>1\right) \leq 2 \sum_{i=1}^{n} \mathbb{P}\left(\left|\xi_{i}\right|>\frac{z}{4}\right)+e^{2}\left(1+\frac{z^{2}}{8}\right)^{-2} \beta_{2} .
$$

For a bound of type (10), we bound $\left(1+\frac{z^{2}}{8}\right)^{-2}$ by $4(1+z)^{-2}$. Thus from (36) and (37),

$$
\begin{aligned}
|\mathbb{P}(W \geq z)-P(\bar{W}>z)| & \leq 2 \sum_{i=1}^{n} \mathbb{P}\left(\left|\xi_{i}\right|>\frac{z}{4}\right)+e^{2}\left(1+\frac{z^{2}}{8}\right)^{-2} \beta_{2} \\
& \leq 2 \sum_{i=1}^{n} \mathbb{P}\left(\left|\xi_{i}\right|>\frac{z}{4}\right)+4 e^{2}(1+z)^{-2}\left(\beta_{2}+\beta_{3}\right),
\end{aligned}
$$

where for the last inequality we used that $\beta_{3} \geq 0$. Hence, using the triangle inequality, we have for $z>2$,

$$
\begin{aligned}
& |\mathbb{P}(W \leq z)-\Phi(z)| \\
& \leq|\mathbb{P}(W \geq z)-\mathbb{P}(\bar{W}>z)|+|\mathbb{P}(\bar{W}>z)-\Phi(-z)| \\
& \leq 2 \sum_{i=1}^{n} P\left(\left|\xi_{i}\right|>\frac{z}{4}\right)+4 e^{2}(1+z)^{-2}\left(\beta_{2}+\beta_{3}\right)+|\mathbb{P}(\bar{W} \leq z)-\Phi(z)| .
\end{aligned}
$$

Note that for $z>2$, we can bound $e^{-\frac{z}{2}} \leq \frac{16}{e^{1.5}}(1+z)^{-2}$.

Now we claim that for $z>2$,

$$
|\mathbb{P}(\bar{W} \leq z)-\Phi(z)| \leq 7115 e^{-\frac{z}{2}}\left(\beta_{2}+\beta_{3}\right) .
$$

If (38) holds, then for $z>2$, bounding $e^{-\frac{z}{2}} \leq \frac{16}{e^{1.5}}(1+z)^{-2}$, we obtain

$$
\begin{aligned}
& |\mathbb{P}(W \leq z)-\Phi(z)| \\
& \leq 2 \sum_{i=1}^{n} \mathbb{P}\left(\left|\xi_{i}\right|>\frac{z}{4}\right)+\left(4 e^{2}+\frac{16}{e^{1.5}} \times 7115\right)(1+z)^{-2}\left(\beta_{2}+\beta_{3}\right) \\
& \leq 2 \sum_{i=1}^{n} \mathbb{P}\left(\left|\xi_{i}\right|>\frac{1 \vee|z|}{4}\right)+25431(1+|z|)^{-2}\left(\beta_{2}+\beta_{3}\right)
\end{aligned}
$$


which proves the theorem when $\beta_{2}+\beta_{3}<1$ and $z>2$ and therefore completes the proof of Theorem 5.1. So our remaining work is to prove (38).

\section{Proof of (38)}

To prove (38) we use Stein's method as well as properties of the solution $f_{z}$ to the Stein Equation (3). We define the function

$$
\bar{K}_{i}(t)=\mathbb{E}\left[\bar{x}_{i}\left(\mathbb{1}_{0 \leq t \leq \overline{x_{i}}}-\mathbb{1}_{\overline{x_{i} \leq t<0}}\right)\right]
$$

where $\bar{x}_{i}=\xi_{i} \mathbb{1}_{\xi_{i} \leq 1}$. Equation (8.24) in [3] and $\sum_{i=1}^{n} \mathbb{E} \xi_{i}{ }^{2}=1$ give

$$
\sum_{i=1}^{n} \int_{-\infty}^{1} \bar{K}_{i}(t) d t=\sum_{i=1}^{n} \mathbb{E} \bar{x}_{i}{ }^{2}=1-\sum_{i=1}^{n} \mathbb{E}\left[\xi_{i}{ }^{2} \mathbb{1}_{\xi_{i}>1}\right] .
$$

Using the independence between $\bar{W}^{(i)}$ and $\bar{x}_{i}$,

$$
\begin{aligned}
\mathbb{E}\left[\bar{W} f_{z}(\bar{W})\right] & =\sum_{i=1}^{n} \mathbb{E}\left[\bar{x}_{i} f_{z}(\bar{W})\right] \\
& =\sum_{i=1}^{n} \mathbb{E}\left[\bar{x}_{i}\left(f_{z}(\bar{W})-f_{z}\left(\bar{W}^{(i)}\right)\right)\right]+\sum_{i=1}^{n} \mathbb{E} \bar{x}_{i} \mathbb{E}\left[f_{z}\left(\bar{W}^{(i)}\right)\right] \\
& =\sum_{i=1}^{n} \mathbb{E}\left[\bar{x}_{i} \int_{0}^{\bar{x}_{i}} f_{z}^{\prime}\left(\bar{W}^{(i)}+t\right) d t\right]+\sum_{i=1}^{n} \mathbb{E} \bar{x}_{i} \mathbb{E}\left[f_{z}\left(\bar{W}^{(i)}\right)\right] .
\end{aligned}
$$

The first term in (42) can be written as

$$
\begin{aligned}
& \sum_{i=1}^{n} \mathbb{E}\left[\bar{x}_{i} \int_{0}^{\bar{x}_{i}} f_{z}^{\prime}\left(\bar{W}^{(i)}+t\right) d t\right] \\
& =\sum_{i=1}^{n} \mathbb{E} \int_{-\infty}^{1} f_{z}^{\prime}\left(\bar{W}^{(i)}+t\right) \bar{x}_{i} \mathbb{1}_{0 \leq t \leq \overline{x_{i}}} d t-\sum_{i=1}^{n} \mathbb{E} \int_{-\infty}^{1} f_{z}^{\prime}\left(\bar{W}^{(i)}+t\right) \bar{x}_{i} \mathbb{1}_{\bar{x}_{i} \leq t<\text { (o }}(4 \mathrm{~d}) \\
& =\sum_{i=1}^{n} \int_{-\infty}^{1} \mathbb{E}\left[f_{z}^{\prime}\left(\bar{W}^{(i)}+t\right)\right] \bar{K}_{i}(t) d t
\end{aligned}
$$

where the last equality follows from independence. Therefore,

$$
\mathbb{E}\left[\bar{W} f_{z}(\bar{W})\right]=\sum_{i=1}^{n} \int_{-\infty}^{1} \mathbb{E}\left[f_{z}^{\prime}\left(\bar{W}^{(i)}+t\right)\right] \bar{K}_{i}(t) d t+\sum_{i=1}^{n} \mathbb{E} \bar{x}_{i} \mathbb{E}\left[f_{z}\left(\bar{W}^{(i)}\right)\right]
$$


Next we replace $w$ by $\bar{W}$ and take expectations in the Stein Equation (3), together with (41) and (45), to obtain

$$
\begin{aligned}
\mathbb{P}(\bar{W} \leq z)-\Phi(z)= & \mathbb{E}\left[f_{z}^{\prime}(\bar{W})\right]-\mathbb{E}\left[\bar{W} f_{z}(\bar{W})\right] \\
= & \mathbb{E}\left[f_{z}^{\prime}(\bar{W})\right]\left(\sum_{i=1}^{n} \int_{-\infty}^{1} \bar{K}_{i}(t) d t+\sum_{i=1}^{n} \mathbb{E}\left[\xi_{i}^{2} \mathbb{1}_{\xi_{i}>1}\right]\right) \\
= & \sum_{i=1}^{n} \mathbb{E}\left[\xi_{i}{ }^{2} \mathbb{1}_{\xi_{i}>1}\right] E\left[f_{z}^{\prime}(\bar{W})\right] \\
& +\sum_{i=1}^{n} \int_{-\infty}^{1} \mathbb{E}\left[f_{z}^{\prime}\left(\bar{W}^{(i)}+\bar{x}_{i}\right)-f_{z}^{\prime}\left(\bar{W}^{(i)}+t\right)\right] \bar{K}_{i}(t) d t \\
& +\sum_{i=1}^{n} \mathbb{E}\left[\xi_{i} \mathbb{1}_{\xi_{i}>1}\right] E\left[f_{z}\left(\bar{W}^{(i)}\right)\right] \\
= & R_{1}+R_{2}+R_{3} .
\end{aligned}
$$

In order to prove (38), we bound each of $R_{1}$ given in (46), $R_{2}$ given in (47) and $R_{3}$ given in (48) and show that the sum of the three bounds is less than or equal to $7115 e^{-\frac{z}{2}}\left(\beta_{2}+\beta_{3}\right)$ for $z>2$.

Bound for $R_{1}$

For $R_{1}=\sum_{i=1}^{n} \mathbb{E}\left[\xi_{i}^{2} \mathbb{1}_{\xi_{i}>1}\right] \mathbb{E}\left[f_{z}^{\prime}(\bar{W})\right]$, substituting (4) into the Stein Equation (3) gives

$$
\begin{aligned}
f_{z}^{\prime}(w) & =w f_{z}(w)+\mathbb{1}_{w \leq z}-\Phi(z) \\
& = \begin{cases}\left(\sqrt{2 \pi} w e^{w^{2} / 2} \Phi(w)+1\right)(1-\Phi(z)) & \text { if } w \leq z \\
\left(\sqrt{2 \pi} w e^{w^{2} / 2}(1-\Phi(w))-1\right) \Phi(z) & \text { if } w>z\end{cases}
\end{aligned}
$$

Using (5),

$$
\begin{aligned}
\mathbb{E}\left|f_{z}^{\prime}(\bar{W})\right| & =\mathbb{E}\left[\left|f_{z}^{\prime}(\bar{W})\right| \mathbb{1}_{\bar{W} \leq \frac{z}{2}}\right]+\mathbb{E}\left[\left|f_{z}^{\prime}(\bar{W})\right| \mathbb{1}_{\bar{W}>\frac{z}{2}}\right] \\
& \left.=\mathbb{E}\left[\left(\sqrt{2 \pi} w e^{w^{2} / 2} \Phi(w)+1\right)(1-\Phi(z)) \mathbb{1}_{\bar{W} \leq \frac{z}{2}}\right)\right]+\mathbb{E}\left[\left|f_{z}^{\prime}(\bar{W})\right| \mathbb{1}_{\bar{W}>\frac{z}{2}}\right] \\
& \leq\left(\sqrt{2 \pi} \frac{z}{2} e^{z^{2} / 8}+1\right)(1-\Phi(z))+\mathbb{P}\left(\bar{W}>\frac{z}{2}\right) .
\end{aligned}
$$

By Markov's inequality, $\mathbb{P}\left(\bar{W}>\frac{z}{2}\right)=\mathbb{P}\left(e^{\bar{W}}>e^{\frac{z}{2}}\right) \leq e^{-\frac{z}{2}} \mathbb{E}\left[e^{\bar{W}}\right]$. By definition $\bar{x}_{i} \leq 1$, so $\mathbb{E} \bar{x}_{i} \leq 0$ and $\sum_{i=1}^{n} \mathbb{E} \bar{x}_{i}^{2} \leq 1$. Applying Lemma 8.2 in [3] with $\alpha=B=t=1$ gives

$$
\mathbb{E}\left[e^{\bar{W}}\right] \leq \exp (e-1-1)=e^{e-2} .
$$


Again employing the standard normal tail bound (2),

$$
\begin{aligned}
\mathbb{E}\left|f_{z}^{\prime}(\bar{W})\right| & \leq \frac{1}{2} e^{-\frac{3}{8} z^{2}}+\frac{1}{z \sqrt{2 \pi}} e^{-\frac{z^{2}}{2}}+e^{-\frac{z}{2}} e^{e-2} \\
& \leq \frac{1}{2} e^{-\frac{1}{2}} e^{-\frac{z}{2}}+\frac{e^{-1}}{2 \sqrt{2 \pi}} e^{-\frac{z}{2}}+e^{-\frac{z}{2}} e^{e-2}
\end{aligned}
$$

Hence, we have shown that

$$
\begin{aligned}
\left|R_{1}\right| & \leq\left(\frac{1}{2} e^{-\frac{1}{2}}+\frac{e^{-1}}{2 \sqrt{2 \pi}}+e^{e-2}\right) e^{-\frac{z}{2}} \sum_{i=1}^{n} \mathbb{E}\left[\xi_{i}{ }^{2} \mathbb{1}_{\xi_{i}>1}\right] \\
& \leq\left(\frac{1}{2} e^{-\frac{1}{2}}+\frac{e^{-1}}{2 \sqrt{2 \pi}}+e^{e-2}\right) e^{-\frac{z}{2}}\left(\beta_{2}+\beta_{3}\right) .
\end{aligned}
$$

Bound for $R_{2}$

For $R_{2}=\sum_{i=1}^{n} \int_{-\infty}^{1} \mathbb{E}\left[f_{z}^{\prime}\left(\bar{W}^{(i)}+\bar{x}_{i}\right)-f_{z}^{\prime}\left(\bar{W}^{(i)}+t\right)\right] \bar{K}_{i}(t) d t$, we use the Stein Equation (3) to write $R_{2}$ as the sum of two quantities, and then bound them separately;

$$
\begin{aligned}
R_{2}= & \sum_{i=1}^{n} \int_{-\infty}^{1} \mathbb{E}\left[\left(\bar{W}^{(i)}+\bar{x}_{i}\right) f_{z}\left(\bar{W}^{(i)}+\bar{x}_{i}\right)+\mathbb{1}_{\bar{W}^{(i)}+\bar{x}_{i} \leq z}-\Phi(z)\right. \\
& \left.-\left(\bar{W}^{(i)}+t\right) f_{z}\left(\bar{W}^{(i)}+t\right)-\mathbb{1}_{\bar{W}^{(i)}+t \leq z}+\Phi(z)\right] \bar{K}_{i}(t) d t \\
= & R_{21}+R_{22}
\end{aligned}
$$

with

$R_{21}=\sum_{i=1}^{n} \int_{-\infty}^{1} \mathbb{E}\left[\mathbb{1}_{\bar{W}^{(i)}+\bar{x}_{i} \leq z}-\mathbb{1}_{\bar{W}^{(i)}+t \leq z}\right] \bar{K}_{i}(t) d t$
$R_{22}=\sum_{i=1}^{n} \int_{-\infty}^{1} \mathbb{E}\left[\left(\bar{W}^{(i)}+\bar{x}_{i}\right) f_{z}\left(\bar{W}^{(i)}+\bar{x}_{i}\right)-\left(\bar{W}^{(i)}+t\right) f_{z}\left(\bar{W}^{(i)}+t\right)\right] \bar{K}_{i}(t) d t$.

Since the difference between two indicator functions is always less than or equal to one, $R_{21}$ can be bounded by

$$
R_{21} \leq \sum_{i=1}^{n} \int_{-\infty}^{1} \mathbb{E}\left[\mathbb{1}_{\bar{x}_{i} \leq t} P\left(z-t<\bar{W}^{(i)} \leq z-\bar{x}_{i} \mid \bar{x}_{i}\right)\right] \bar{K}_{i}(t) d t
$$


Applying Proposition 8.1 from [3] with $a=z-t$ and $b=z-\bar{x}_{i}$ gives

$$
\begin{aligned}
R_{21} & \leq \sum_{i=1}^{n} \int_{-\infty}^{1} \mathbb{E}\left[6\left(\min \left(1, t-\bar{x}_{i}\right)+\beta_{2}+\beta_{3}\right) e^{-\frac{z-t}{2}}\right] \bar{K}_{i}(t) d t \\
& \leq 6 e^{-\frac{z}{2}} e^{\frac{1}{2}} \sum_{i=1}^{n} \int_{-\infty}^{1} \mathbb{E}\left[\min \left(1,|t|+\left|\bar{x}_{i}\right|\right)+\beta_{2}+\beta_{3}\right] \bar{K}_{i}(t) d t \\
& \leq 6 e^{-\frac{z}{2}} e^{\frac{1}{2}}\left(\beta_{2}+\beta_{3}\right)+6 e^{-\frac{z}{2}} e^{\frac{1}{2}} \sum_{i=1}^{n} \int_{-\infty}^{1} \mathbb{E}\left[\min \left(1,|t|+\left|\bar{x}_{i}\right|\right)\right] \bar{K}_{i}(t) d t
\end{aligned}
$$

where we used (41) for the last step. Note that $\mathbb{1}_{0 \leq t \leq \overline{x_{i}}}+\mathbb{1}_{\bar{x}_{i} \leq t<0} \leq \mathbb{1}_{|t| \leq\left|\bar{x}_{i}\right|}$, so $\bar{K}_{i}(t) \leq \mathbb{E}\left[\left|\bar{x}_{i}\right| \mathbb{1}_{|t| \leq\left|\bar{x}_{i}\right|}\right]$. Moreover, as both $\min \left(\overline{1},|t|+\left|\bar{x}_{i}\right|\right)$ and $\left|\bar{x}_{i}\right| \mathbb{1}_{|t| \leq\left|\bar{x}_{i}\right|}$ are increasing functions of $\left|\bar{x}_{i}\right|$, they are positively correlated. So

$$
\begin{aligned}
\mathbb{E}\left[\min \left(1,|t|+\left|\bar{x}_{i}\right|\right)\right] \bar{K}_{i}(t) & \leq \mathbb{E}\left[\min \left(1,|t|+\left|\bar{x}_{i}\right|\right)\right] \mathbb{E}\left[\left|\bar{x}_{i}\right| \mathbb{1}_{|t| \leq\left|\bar{x}_{i}\right|}\right] \\
& \leq \mathbb{E}\left[\min \left(1,|t|+\left|\overline{x_{i}}\right|\right)\left|\overline{x_{i}}\right| \mathbb{1}_{|t| \leq\left|\bar{x}_{i}\right|}\right] \\
& \leq 2 \mathbb{E}\left[\min \left(1,\left|\bar{x}_{i}\right|\right)\left|\bar{x}_{i}\right| \mathbb{1}_{|t| \leq\left|\bar{x}_{i}\right|}\right]
\end{aligned}
$$

This gives

$$
\begin{aligned}
& \sum_{i=1}^{n} \int_{-\infty}^{1} \mathbb{E}\left[\min \left(1,|t|+\left|\bar{x}_{i}\right|\right)\right] \bar{K}_{i}(t) d t \\
& \leq \sum_{i=1}^{n} \int_{-\infty}^{1} 2 \mathbb{E}\left[\min \left(1,\left|\bar{x}_{i}\right|\right)\left|\bar{x}_{i}\right| \mathbb{1}_{|t| \leq\left|\bar{x}_{i}\right|}\right] d t \\
& \leq 4 \sum_{i=1}^{n} \mathbb{E}\left[\min \left(1,\left|\bar{\xi}_{i}\right|\right)\left|\bar{\xi}_{i}\right|^{2}\right]=4\left(\beta_{2}+\beta_{3}\right) .
\end{aligned}
$$

Substituting (53) into (51),

$$
R_{21} \leq 6 e^{-\frac{z}{2}} e^{\frac{1}{2}}(4+1)\left(\beta_{2}+\beta_{3}\right)=30 e^{\frac{1}{2}}\left(\beta_{2}+\beta_{3}\right) e^{-\frac{z}{2}} .
$$

Similarly, we can construct a lower bound for $R_{21}$ by symmetry,

$$
\begin{aligned}
R_{21} & \geq \sum_{i=1}^{n} \int_{-\infty}^{1} \mathbb{E}\left[-\mathbb{1}_{t \leq \overline{x_{i}}} P\left(z-\bar{x}_{i}<\bar{W}^{(i)} \leq z-t \mid \bar{x}_{i}\right)\right] \bar{K}_{i}(t) d t \\
& \geq-\sum_{i=1}^{n} \int_{-\infty}^{1} \mathbb{E}\left[6\left(\min \left(1, \bar{x}_{i}-t\right)+\beta_{2}+\beta_{3}\right) e^{-\frac{z-\bar{x}_{i}}{2}}\right] \bar{K}_{i}(t) d t \\
& \geq-6 e^{-\frac{z}{2}} e^{\frac{1}{2}} \sum_{i=1}^{n} \int_{-\infty}^{1} \mathbb{E}\left[\min \left(1,|t|+\left|\bar{x}_{i}\right|\right)+\beta_{2}+\beta_{3}\right] \bar{K}_{i}(t) d t
\end{aligned}
$$


Proceeding now as for (53) gives that $R_{21} \geq-30 e^{\frac{1}{2}}\left(\beta_{2}+\beta_{3}\right) e^{-\frac{z}{2}}$ and therefore,

$$
\left|R_{21}\right| \leq 30 e^{\frac{1}{2}}\left(\beta_{2}+\beta_{3}\right) e^{-\frac{z}{2}} .
$$

For $R_{22}$, since $w f_{z}(w)$ is increasing in $w$, Lemma 4.1 gives

$$
\begin{aligned}
R_{22} \leq & \sum_{i=1}^{n} \int_{-\infty}^{1} \mathbb{E}\left[\mathbb{1}_{t \leq \bar{x}_{i}}\left(\bar{W}^{(i)}+\bar{x}_{i}\right) f_{z}\left(\bar{W}^{(i)}+\bar{x}_{i}\right) \mid \bar{x}_{i}\right. \\
& \left.-\left(\bar{W}^{(i)}+t\right) f_{z}\left(\bar{W}^{(i)}+t\right)\right] \bar{K}_{i}(t) d t \\
\leq & \left(25.8+\frac{20 e^{e^{2}-2}}{\sqrt{2 \pi}}\right) e^{-\frac{z}{2}} \sum_{i=1}^{n} \int_{-\infty}^{1} \mathbb{E}\left[\min \left(1,\left|\bar{x}_{i}\right|+|t|\right)\right] \bar{K}_{i}(t) d t \\
\leq & \left(103.2+\frac{80}{\sqrt{2 \pi}} e^{e^{2}-2}\right) e^{-\frac{z}{2}}\left(\beta_{2}+\beta_{3}\right) . .
\end{aligned}
$$

Here we used (53) for the last step. A lower bound for $R_{22}$ follows similarly,

$$
\begin{gathered}
R_{22} \geq \sum_{i=1}^{n} \int_{-\infty}^{1} \mathbb{E}\left[\mathbb{1}_{\bar{x}_{i} \leq t}\left(\bar{W}^{(i)}+\bar{x}_{i}\right) f_{z}\left(\bar{W}^{(i)}+\bar{x}_{i}\right) \mid \bar{x}_{i}\right. \\
\left.-\left(\bar{W}^{(i)}+t\right) f_{z}\left(\bar{W}^{(i)}+t\right)\right] \bar{K}_{i}(t) d t \\
\geq-\sum_{i=1}^{n} \int_{-\infty}^{1} \mathbb{E}\left[\mathbb{1}_{\overline{x_{i}} \leq t}\left(\bar{W}^{(i)}+t\right) f_{z}\left(\bar{W}^{(i)}+t\right)\right. \\
\left.-\left(\bar{W}^{(i)}+\bar{x}_{i}\right) f_{z}\left(\bar{W}^{(i)}+\bar{x}_{i}\right) \mid \bar{x}_{i}\right] \bar{K}_{i}(t) d t \\
\geq-\left(103.2+\frac{80}{\sqrt{2 \pi}} e^{e^{2}-2}\right) e^{-\frac{z}{2}}\left(\beta_{2}+\beta_{3}\right) .
\end{gathered}
$$

Collecting (56), (57) and (58) gives

$$
\left|R_{2}\right| \leq\left|R_{21}\right|+\left|R_{22}\right| \leq\left(30 e^{\frac{1}{2}}+103.2+\frac{80}{\sqrt{2 \pi}} e^{e^{2}-2}\right) e^{-\frac{z}{2}}\left(\beta_{2}+\beta_{3}\right) .
$$

Bound for $R_{3}$

Finally, for $R_{3}=\sum_{i=1}^{n} \mathbb{E}\left[\xi_{i} \mathbb{1}_{\xi_{i}>1}\right] \mathbb{E}\left[f_{z}\left(\bar{W}^{(i)}\right)\right]$, we use similar arguments as for $R_{1}$;

$$
\begin{aligned}
\mathbb{E}\left|f_{z}\left(\bar{W}^{(i)}\right)\right| & =\mathbb{E}\left[\left|f_{z}\left(\bar{W}^{(i)}\right)\right| \mathbb{1}_{\bar{W}^{(i)} \leq \frac{z}{2}}\right]+\mathbb{E}\left[\left|f_{z}\left(\bar{W}^{(i)}\right)\right| \mathbb{1}_{\bar{W}^{(i)}>\frac{z}{2}}\right] \\
& \leq \sqrt{2 \pi} e^{\frac{z^{2}}{8}}(1-\Phi(z))+\mathbb{E}\left[\left|f_{z}\left(\bar{W}^{(i)}\right)\right| \mathbb{1}_{\bar{W}^{(i)}>\frac{z}{2}}\right] .
\end{aligned}
$$


From ([6) $), 0<f_{z} \leq \min \left(\frac{\sqrt{2 \pi}}{4}, \frac{1}{|z|}\right)=\frac{1}{|z|} \leq \frac{1}{2}$ for $z>2$. The standard normal tail bound (2) and Lemma 8.2 in [3] with $\alpha=B=t=1$ give

$$
\begin{aligned}
\mathbb{E}\left|f_{z}\left(\bar{W}^{(i)}\right)\right| & \leq \sqrt{2 \pi} e^{\frac{z^{2}}{8}} \frac{1}{z \sqrt{2 \pi}} e^{-\frac{z^{2}}{2}}+\frac{1}{2} P\left(\bar{W}^{(i)}>\frac{z}{2}\right) \\
& \leq \frac{1}{z} e^{-\frac{3}{8} z^{2}}+\frac{1}{2} e^{-\frac{z}{2}} e^{e-2} \\
& \leq \frac{1}{2} e^{-\frac{1}{2}} e^{-\frac{z}{2}}+\frac{1}{2} e^{-\frac{z}{2}} e^{e-2} .
\end{aligned}
$$

Hence, we have shown that

$$
\begin{aligned}
\mid R_{3} & \leq \frac{1}{2}\left(e^{-\frac{1}{2}}+e^{e-2}\right) e^{-\frac{z}{2}} \sum_{i=1}^{n} \mathbb{E}\left[\xi_{i} \mathbb{1}_{\xi_{i}>1}\right] \\
& \leq \frac{1}{2}\left(e^{-\frac{1}{2}}+e^{e-2}\right) e^{-\frac{z}{2}}\left(\beta_{2}+\beta_{3}\right) .
\end{aligned}
$$

Applying (49), (59) and (60) to (46), (47) and (48) respectively, we have

$$
\begin{aligned}
& |P(\bar{W} \leq z)-\Phi(z)| \\
& \leq\left(31 e^{-\frac{1}{2}}+\frac{3}{2} e^{e-2}+103.2+\frac{0.5 e^{-1}+80 e^{e^{2}-2}}{\sqrt{2 \pi}}\right) e^{-\frac{z}{2}}\left(\beta_{2}+\beta_{3}\right) \\
& \leq 7115 e^{-\frac{z}{2}}\left(\beta_{2}+\beta_{3}\right) .
\end{aligned}
$$

This completes the proof of (38) and therefore the proof of (39). Thus we have proved the theorem when $\beta_{2}+\beta_{3}<1$ and $z>2$. Hence we have proved Theorem 3.3 .

\section{Appendix: Results from [3]}

For convenience here we give some results from [3] which we use in this paper.

Let $\xi_{1}, \ldots, \xi_{n}$ denote independent random variables with zero means and variances summing to one. Let $W$ denote their sum, $W=\sum_{i=1}^{n} \xi_{i}$. We consider the truncated random variables and their sums

$$
\bar{x}_{i}=\xi_{i} \mathbb{1}\left(\xi_{i} \leq 1\right), \quad \bar{W}=\sum_{i=1}^{n} \bar{x}_{i}, \text { and } \bar{W}^{(i)}=\bar{W}-\bar{x}_{i} .
$$

In [3], Proposition 8.1, it is shown that for all real $a<b$ and $i=1, \ldots, n$,

$$
\mathbb{P}\left(a \leq \bar{W}^{(i)} \leq b\right) \leq 6\left(\min (1, b-a)+\beta_{2}+\beta_{3}\right) e^{-\frac{a}{2}} .
$$

Moreover, Lemmas 8.1 and 8.2 in [3] gives the next result. 
Lemma .2. [Lemmas 8.1 and 8.2] Let $\eta_{1}, \ldots, \eta_{n}$ be independent random variables satisfying $E \eta_{i} \leq 0$ for $1 \leq i \leq n$ and $\sum_{i=1}^{n} E \eta_{i}^{2} \leq B^{2}$. Then for $x>0$ and $p \geq 1$, with $S_{n}=\sum_{i=1}^{n} \eta_{i}$,

$$
\mathbb{P}\left(S_{n} \geq x\right) \leq P\left(\max _{1 \leq i \leq n} \eta_{i}>\frac{x \vee B}{p}\right)+e^{p}\left(1+\frac{x^{2}}{p B^{2}}\right)^{-p} .
$$

If moreover, for some $\alpha>0, \eta_{i} \leq \alpha$ for all $1 \leq i \leq n$, then, for $t>0$,

$$
\mathbb{E} e^{t S_{n}} \leq \exp \left(\alpha^{-2}\left(e^{t \alpha}-1-t \alpha\right) B^{2}\right) .
$$

Lemma 8.3 in [3] with $t=1$ gives the next result.

Lemma .3. [Lemmas 8.3] Let $\xi_{1}, \ldots, \xi_{n}$ be independent random variables with zero means and variances summing to one. Let $W=\sum_{i=1}^{n} \xi_{i}$ and $\beta_{2}$ be given as above. Then for $z \geq 2$ and $p \geq 2$,

$$
\mathbb{P}\left(W \geq z, \max _{1 \leq i \leq n} \xi_{i}>1\right) \leq 2 \sum_{i=1}^{n} \mathbb{P}\left(\left|\xi_{i}\right|>\frac{z}{2 p}\right)+e^{p}\left(1+\frac{z^{2}}{4 p}\right)^{-p} \beta_{2} .
$$

Acknowledgement. The authors would like to thank Aihua Xia (Melbourne) for helpful discussion.

\section{References}

[1] Ahmad, I. A. (1981). The exact order of normal approximation in bivariate renewal theory. Advances in Applied Probability, 13(1), 113128.

[2] Billingsley, P. (1968). Convergence of Probability Measures. John Wiley \& Sons, New York, London, Sydney, Toronto.

[3] Chen. L.H.Y, Goldstein. L, And Shao, Q.-M. (2011). Normal approximation by Stein's method.Springer, Heidelberg.

[4] Chen, L. H. Y., \& Shao, Q. M. (2001). A non-uniform BerryEsseen bound via Stein's method. Probability Theory and Related Fields, 120(2), 236-254.

[5] Chen, L. H., \& Shao, Q. M. (2005). Steins method for normal approximation. In: An introduction to Steins method, A.D. Barbour and L.H.Y. Chen eds, Singapore University Press and World Scientific, 159. 
[6] Englund, G. (1980). Remainder term estimate for the asymptotic normality of the number of renewals. Journal of Applied Probability, 17(4), 1108-1113.

[7] Niculescu, S. P., \& Omey, E. (1994). Asymptotic behaviour of the distribution function of multivariate nonhomogeneous renewal processes. Studia Scientiarum Mathematicarum Hungarica, 29(1), 179-190.

[8] Omey, E., \& Vesilo, R. (2016). Local limit theorems for shock models. Brazilian Journal of Probability and Statistics, 30(2), 221-247.

[9] Roginsky, A. L. (1992). A central limit theorem by remainder term for renewal processes. Advances in Applied Probability, 24(2), 267-287.

[10] Stein, C. (1972). A bound for the error in the normal approximation to the distribution of a sum of dependent random variables. In Proceedings of the Sixth Berkeley Symposium on Mathematical Statistics and Probability, Volume 2: Probability Theory. The Regents of the University of California. 